\title{
Cardiovascular risk assessment in patients with a severe mental illness: a systematic review and meta-analysis
}

Quintí Foguet-Boreu 1,2*, Maria Isabel Fernandez San Martin 3,1, Gemma Flores Mateo ${ }^{1}$, Edurne Zabaleta del Olmo ${ }^{1,4}$, Luís Ayerbe García-Morzon ${ }^{5,6}$, Maria Perez-Piñar López ${ }^{5}$, Luis Miguel Martin-López , Javier Montes Hidalgo ${ }^{8}$ and Concepción Violán ${ }^{1}$

\begin{abstract}
Background: Cardiovascular risk (CVR) has been observed to be higher in patients with severe mental illness (SMI) than in the general population. However, some studies suggest that CVR is not equally increased in different subgroups of SMI. The purposes of this review are to summarise CVR scores of SMI patients and to determine the differences in CVR between patients with different SMIs and between SMI patients and the control-population.

Methods: MEDLINE (via PubMed) was searched for literature published through August 28, 2014, followed by a snowball search in the Web of Science. Observational and experimental studies that reported CVR assessments in SMI patients using validated tools were included. The risk of bias was reported using STROBE and CONSORT criteria. Pooled continuous data were expressed as standardized mean differences (SMD) with $95 \%$ confidence intervals (CI). Two reviewers independently selected studies, extracted data and assessed methodological quality.

Results: A total of 3,608 articles were identified, of which 67 full text papers were assessed for eligibility and 35 were finally included in our review, in which 12,179 psychiatric patients and 225,951 comparative patients had been assessed. The most frequent diagnoses were schizophrenia and related diagnoses (45.7\%), depressive disorders (14.7\%), SMI (11.4\%) and bipolar disorders (8.6\%). The most frequent CVR assessment tool used was the Framingham risk score. Subgroups analysis showed a higher CVR in schizophrenia than in depressive disorder or in studies that included patients with multiple psychiatric diagnoses (SMD: 0.63, 0.03, and 0.02, respectively). Six studies were included in the meta-analysis. Total overall CVR did not differ between SMI patients and controls (SMD: 0.35 [95 \% Cl:-0.02 to 0.71], $p=0.06)$; high heterogeneity was observed $\left(l^{2}=93 \% ; p<0.001\right)$.

Conclusions: The summary of results from studies that assessed CVR using validated tools in SMI patients did not find sufficient data (except for limited evidence associated with schizophrenia) to permit any clear conclusions about increased CVR in this group of patients compared to the general population.

The systematic review is registered in PROSPERO: CRD42013003898.
\end{abstract}

Keywords: Cardiovascular risk, Severe mental illness, Depressive disorder, Bipolar disorder, Schizophrenia, Systematic review

\footnotetext{
* Correspondence: qfoguet@idiapjgol.org; 42292qfb@comb.cat

${ }^{1}$ Institut Universitari d'Investigació en Atenció Primària Jordi Gol (IDIAP Jordi

Gol), Universitat Autònoma de Barcelona, Gran Via Corts Catalanes, 587 àtic,

08007 Barcelona, Spain

${ }^{2}$ Department of Medical Sciences, School of Medicine, University of Girona,

Emili Grahit, 77, 17071 Girona, Spain

Full list of author information is available at the end of the article
} 


\section{Background}

Cardiovascular disease is the leading cause of overall mortality, accounting for $24 \%$ of deaths worldwide, while psychiatric diseases, led by major depressive disorder, are considered the eleventh most burdensome disease globally, with an increasing effect on overall mortality $[1,2]$.

Criteria for the definition of severe mental illness (SMI) differ, with some authors applying a narrow definition based on psychosis [3] and others also including a set of nosological entities of different types and clinical symptoms but with several common diagnostic criteria: severity, persistence over time (2 years or more), and a tendency toward clinical deterioration and difficulties in social and occupational function $[4,5]$.

It has been reported that cardiovascular risk (CVR) is higher in patients with SMI [6]. Studies in patients with bipolar disorder and schizophrenia indicate that they have a higher CVR than in the general population [7]. In patients with schizophrenia, the most prevalent CVR factors are hyperlipidaemia (61 \%), smoking (55\%), obesity (41\%), diabetes (19\%) and hypertension (17\%)) [8]. Risk of metabolic syndrome is also higher among patients with schizophrenia and bipolar disorder [9]. Moreover, patients with anxiety and major depression have higher prevalence of hypertension compared to groups of similar age from the general population $[10,11]$.

Several factors may contribute to this raised CVR among patients with SMI, including unhealthy behaviours, difficulties in communication, barriers to medical care, poor treatment adherence and social deprivation [12]. Patients with SMI often receive fragmented medical care and fewer preventive measures, which leads to higher levels of underdiagnosis and lower rates of disease control [13]. Furthermore, antipsychotic drugs, antidepressants, and mood-stabilizing drugs have deleterious side effects, including important cardiometabolic consequences [14-16].

However, to date no systematic analysis has investigated whether CVR is increased equally in all patients with SMI, making it difficult to design and implement effective, feasible, evidence-based interventions for CVR management in these patients. A summary of the observations about CVR in the different subgroups of patients with SMI would provide a better epidemiological description of the problem, inform more effective clinical and preventive strategies and help in the design of further studies.

The major aim of this review was to summarize the available evidence of CVR scores in patients with SMI. Furthermore, this review attempted to determine whether CVR differs between subgroups of SMI patients and compare the CVR between patients with SMI and the general or non-psychiatric population.

\section{Methods}

The Meta-analysis Of Observational Studies in Epidemiology (MOOSE) criteria were used to undertake this review and meta-analysis [17], together with Preferred Reporting Items for Systematic Reviews and Meta-Analyses (PRISMA) [18]. We conducted a systematic review of studies that reported CVR in patients with SMI.

\section{Eligibility criteria}

We included studies that reported CVR scores in patients with SMI. The following 10 diagnoses were included in the search strategy (Table 1): schizophrenic disorders, schizotypal disorders, persistent delirious disorders, induced delirious disorders, schizoaffective disorders, other non-organic psychotic disorders, bipolar disorder, serious depressive episode with psychotic symptoms, recurrent serious depressive disorders, and compulsive obsessive disorder [5].

We included observational and experimental studies that applied validated CVR tools, including Framingham risk score (FRS) with its subtypes of scores (cardiovascular disease (CVD), cardiovascular heart disease (CHD), Myocardial infarction (MI) and the Systematic Coronary Risk Evaluation (SCORE). If the studies reported data on other CVR scores not described above, these were also included.

We excluded articles that were based on first episodes of SMI, different reports from the same population

Table 1 Search strategies for the electronic databases (data retrieved August 28, 2014)

\begin{tabular}{ll}
\hline Database & Search Strategy \\
\hline PubMed & ("Psychotic Disorders"[Mesh] OR "Bipolar Disorder"[Mesh] OR "Schizophrenia"[Mesh] OR psychotic OR psychosis \\
& OR psychoses OR schizo* OR bipolar OR manic OR mania OR delirious OR depress* OR obsessive-compulsive OR \\
& "obsessive compulsive" OR "compulsive obsessive" OR OCD OR agoraphob* OR panic OR phobia OR phobic OR \\
& melancho* OR neurosis OR neurotic OR neuroses OR conversion disorder* OR "Mental Disorders" OR "severe \\
& mental") AND (cardiovascular OR "Cardiovascular diseases" OR CVD) AND (risk score* OR risk chart* OR "risk \\
& prediction" OR risk check* OR "risk assessment" OR "risk evaluation" OR "risk calculator" OR risk-estimation OR \\
& "risk estimation" OR "year risk" OR "year CVD risk" OR Framingham OR "SCORE risk" OR SCORE chart* OR SCORE \\
& table*OR "Systematic Coronary Risk Evaluation" OR "REGICOR" OR "REGICOR table* OR ASSIGN OR QRISK OR \\
& PROCAM OR WHO/ISH)
\end{tabular}


(selecting the study with the most recent publication date or the largest sample size), papers reporting diagnoses based on symptoms, and studies referring to one or two psychotropic drugs.

\section{Search strategy}

We conducted a systematic search in PubMed using a combination of MESH and free text terms (Table 1). We searched from inception to the August 28, 2014. Based on the articles selected, we performed a snowball search in the Web of Science. We reviewed all the references (backward search) and the articles that cited the included papers (forward search). In addition, we added articles that were identified during the implementation of the review (hand searching). There were no language restrictions.

\section{Study selection}

Two researchers (CVF and QFB) reviewed the titles and abstracts of all studies identified in the initial search and defined a list of full text articles to be assessed. Cases of discordance were resolved by consensus; when necessary, the full-text article was reviewed. We conducted a pilot test of the eligibility criteria on a sample of 15 articles. We used this test to clarify these criteria and ensure that they were applied consistently by all reviewers.

Primary outcome was the CVR assessed with any validated CVR tool.

\section{Data collection}

We used a standardized data-collection form to record author and publication year, study design, country, setting, diagnosis, diagnostic criteria, number of participants and age in the psychiatric group and the comparative group (if applicable) and the objective of the study.

To assess the methodological quality of the studies, we used the Strengthening the Reporting of Observational Studies in Epidemiology Statement (STROBE) checklist for observational studies, with a maximum possible score of 24 [19], and the Consolidated Standards of Reporting Trials (CONSORT) for randomized trials, with a maximum possible score of 37 [20], giving one point for each item the article addressed.

Two reviewers assessed methodological quality and extracted the data independently. Discrepancies were resolved by consensus between the two reviewers (CVV and QFB) and by discussion with a third reviewer (MFS) as needed. Inter-rater agreement was $96 \%$.

\section{Statistical analysis}

We analysed outcomes using Review Manager (RevMan, version 5.3). Pooled continuous data were expressed as standardized mean differences (SMD) with $95 \%$ confidence intervals $(\mathrm{CI})$. The effect size (ES) was categorized as small $(<0.2)$, small to moderate $(0.2-0.5)$, moderate to large (0.51-0.79), large $(>0.79)$. Pooled SMD were estimated by using an inverse-variance-weighted randomeffects model. Heterogeneity was quantified with the $\mathrm{I}^{2}$ statistic, which describes the proportion of the total between-study variability due to heterogeneity [21]. We used subgroup analysis to evaluate whether results differed according to the diagnosis (depressive disorder, schizophrenia vs psychiatric diagnoses), diagnosis criteria (non-specific (NE) vs DSM IV), study design (observational vs randomized control trial); and outcome (cardiovascular disease, coronary heart disease, stroke)

We assessed publication bias by using funnel plots. In sensitivity analysis, we assessed the relative influence of each study on the pooled estimate by omitting one study at time.

\section{Protocol and registration}

The initial protocol of the review was submitted to the International Prospective Register of Systematic Reviews (PROSPERO, http://www.crd.york.ac.uk/PROSPERO/). The definitive protocol included the modifications suggested by the PROSPERO reviewers. The registration number of systematic review is: CRD42013003898.

\section{Results}

The electronic and manual searches retrieved 3,608 articles, of which 67 full-text papers were assessed for eligibility and 35 studies were finally included in our review (Fig. 1), representing a total of 12,179 psychiatric patients and 225,951 controls. Sample size of psychiatric study groups ranged from 36 [22] to 1,942 [23] participants. Of the 35 studies, 19 studies in Eurasia (16 in Europe) and 16 studies were conducted in the Americas. The most common design was cross-sectional (22 studies); 8 studies were randomized controlled trials (RCT) and only 5 were case-control studies (Table 2). A $45.7 \%$ of the studies were performed in secondary services exclusively and $31.4 \%$ in the hospital setting. The most frequent diagnoses were schizophrenia and related diagnoses (45.7\%), depressive disorders (14.3\%), and bipolar disorders $(8.6 \%)$. Seven studies $(20.0 \%)$ included different psychiatric diagnoses and only 4 (11.4\%) showed data on SMI as a whole (Table 1).

In 30 studies, methodological quality was evaluated with STROBE and most showed a high quality score (median 21.00, SD: 6.40). Five were evaluated with CONSORT and most had a low quality score (median 18.57, SD: 2.72). The STROBE evaluation revealed two main weaknesses: insufficient efforts to address potential sources of bias and sparse information for each variable of interest on the number of participants with missing data. The CONSORT weaknesses were the method used to generate the random allocation sequence and type of 


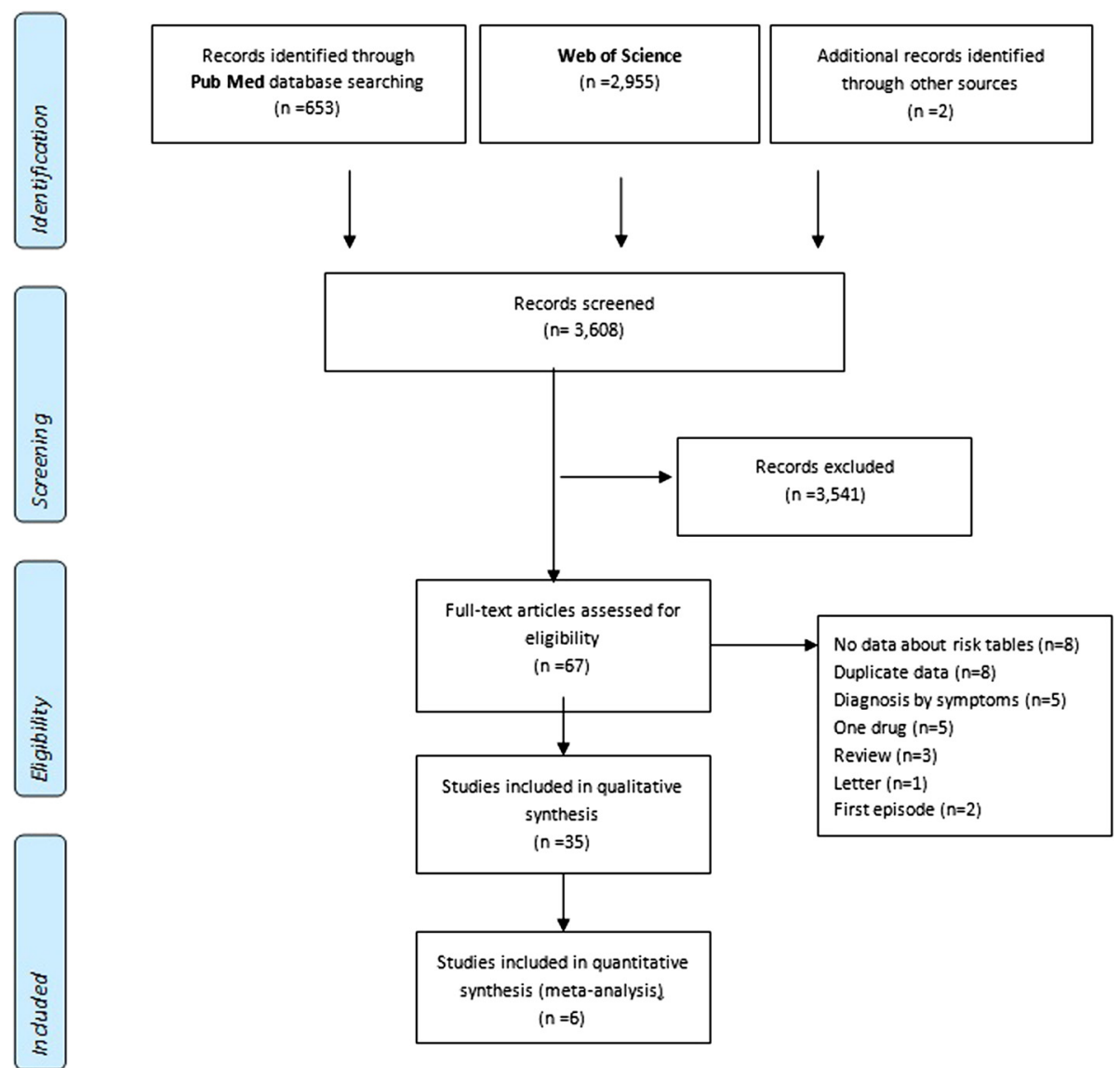

Fig. 1 Flow diagram of article review process

randomisation; details of any restriction (such as blocking and block size); and information about where the full trial protocol can be accessed, if available (Additional file 1: Appendix 1).

Of the 35 studies included, only 7 studies had control groups [22, 24-29]. These studies used different scores to evaluate CVR (Table 2). Three studies included only patients with schizophrenia and controls: two studies were based on FRS (CVD) scores: 10.7 vs. $8.5 p \leq 0.01$ [28] and 4.7 (4.7) vs. 3.1 (3.2), $p=0.002$ [29] and one was based on FRS (CHD) scores: 8.6 (7.3) vs. 6.3 (6.0), $p<0.001$ [24]. Two studies included psychiatric diagnoses and were based on FRS (CVD) scores: $11.3(12.3)$ vs. 6.8 (6.4), $p<0.01$ [25] and 8.3 (5.8) vs. 10.7 (5.9), $p=0.05$ [26]. One study included depressive disorders: 10.3 (7.6) vs. 10.1 (7.7), $p=0.97$ [22]. One study had insufficient data and was not included in the meta-analysis.

Table 3 synthesized the data about CVR scores found in studies by diagnosis groups of diseases. The CVR mean score assessed with FRS (CVD) in patients with depression ranged from 5.8 to 14.0, in patients with schizophrenia from 4.7 to 11.9 , and was 13.7 in the only study of patients with bipolar disorder. Studies that addressed patients with SMI reported that CVR had been expressed in different forms (Table 3).

Subgroup analysis was performed in six studies (3 involving schizophrenia, 1 depressive disorder and 2 psychiatric diagnoses in general). Higher CVR was observed in patients with schizophrenia than in those with depressive disorder or general psychiatric diagnosis, with a pooled SMD (95 \% CI) as follows: $0.63(0.16$, $1.09), 0.03(-0.48,0.54)$, and $0.02(-0.82,0.86)$, respectively (Table 4$)$. The sensitivity analysis omitted one study at a time, showing a pooled SMD ranging from 0.19 to 0.50 . Funnel plots did not suggest any publication bias (Additional file 2: Appendix 2).

Six studies that included 1,065 people with SMI who had a CVR assessment and 1,567 people without SMI were included in the meta-analysis [22, 24-27, 29]. The total overall CVR between the psychiatric group and control group showed a SMD of 0.35 (95 \% CI:-0.02-0.71, $P=0.06)$ with significantly high heterogeneity $\left(I^{2}=93 \% ; p<0.001\right)$ (Fig. 2). 
Table 2 Characteristics of the studies included in the review

\begin{tabular}{|c|c|c|c|c|c|c|c|c|c|c|c|c|c|c|c|c|}
\hline \multirow[t]{3}{*}{ Author, year } & \multirow[t]{3}{*}{ Study design } & \multirow[t]{3}{*}{ Country } & \multirow[t]{3}{*}{ Setting } & \multirow[t]{3}{*}{ Diagnosis } & \multirow{3}{*}{$\begin{array}{l}\text { Diagnostic } \\
\text { criteria }\end{array}$} & \multicolumn{6}{|c|}{ Psychiatric Group } & \multicolumn{5}{|c|}{ Comparative Group } \\
\hline & & & & & & \multirow[t]{2}{*}{ Number } & \multirow{2}{*}{$\begin{array}{l}\text { Age } \\
\text { [mean(SD)] }\end{array}$} & \multicolumn{3}{|c|}{ FRS [mean (SD)] } & \multirow{2}{*}{$\begin{array}{l}\text { SCORE } \\
\text { [mean (SD)] }\end{array}$} & \multirow[t]{2}{*}{ Number } & \multirow{2}{*}{$\begin{array}{l}\text { Age } \\
\text { [mean(SD)] }\end{array}$} & \multicolumn{3}{|c|}{ FRS [mean (SD)] } \\
\hline & & & & & & & & $\overline{C V D}$ & Stroke & CHD & & & & CVD & Stroke & CHD \\
\hline $\begin{array}{l}\text { Acharya T, } \\
2013 \text { [43] }\end{array}$ & $\begin{array}{l}\text { Retrospective } \\
\text { cross- } \\
\text { sectional }\end{array}$ & USA & Hospital & $\begin{array}{l}\text { Depressive } \\
\text { disorder }\end{array}$ & $\begin{array}{l}\text { Not } \\
\text { reported }\end{array}$ & 1.136 & $60.1(3.0)$ & & & By drug & & 472 & $61,4(11.9)$ & & & $17.1(5.7)$ \\
\hline $\begin{array}{l}\text { Allan CL, } \\
2011[22]\end{array}$ & $\begin{array}{l}\text { Cross- } \\
\text { sectional }\end{array}$ & UK & $\begin{array}{l}\text { Primary \& } \\
\text { secondary } \\
\text { care services }\end{array}$ & $\begin{array}{l}\text { Depressive } \\
\text { disorder }\end{array}$ & DSM IV & 36 & $71.8(7.7)$ & & $10.3(7.6)$ & & & 25 & $71.8(7.3)$ & & $10.1(7.7)$ & \\
\hline $\begin{array}{l}\text { Arango C, } \\
2008[44]\end{array}$ & $\begin{array}{l}\text { Cross- } \\
\text { sectional }\end{array}$ & Spain & $\begin{array}{l}\text { Secondary } \\
\text { services }\end{array}$ & $\begin{array}{l}\text { Schizophrenia \& } \\
\text { related disorders }\end{array}$ & DSM IV & 1.452 & $40.7(12.2)$ & & & $6.8(6.9)$ & $0.9(1.9)$ & & & & & \\
\hline $\begin{array}{l}\text { Bernardo M, } \\
2009 \text { [45] }\end{array}$ & $\begin{array}{l}\text { Cross- } \\
\text { sectional }\end{array}$ & Spain & $\begin{array}{l}\text { Psychiatric } \\
\text { hospital }\end{array}$ & Schizophrenia & DSM IV & 733 & $37.8(11.3)$ & & & & & & & & & \\
\hline $\begin{array}{l}\text { Cohn T, } \\
2004 \text { [37] }\end{array}$ & $\begin{array}{l}\text { Cross- } \\
\text { sectional }\end{array}$ & Canada & $\begin{array}{l}\text { Psychiatric } \\
\text { hospital \& } \\
\text { secondary } \\
\text { services }\end{array}$ & $\begin{array}{l}\text { Schizophrenia \& } \\
\text { related disorders }\end{array}$ & DSM IV & 240 & $43.6(1.3)$ & & & & & 7,020 & $43.6(1.3)$ & & & \\
\hline $\begin{array}{l}\text { Correll CU, } \\
2006[46]\end{array}$ & $\begin{array}{l}\text { Cross- } \\
\text { sectional }\end{array}$ & USA & $\begin{array}{l}\text { Psychiatric } \\
\text { hospital }\end{array}$ & $\begin{array}{l}\text { Psychiatric } \\
\text { diagnosis }\end{array}$ & $\begin{array}{l}\text { Not } \\
\text { reported }\end{array}$ & 367 & $42.9(15.3)$ & & & & & & & & & \\
\hline $\begin{array}{l}\text { Correll CU, } \\
2011 \text { [47] }\end{array}$ & $\begin{array}{l}\text { Cross- } \\
\text { sectional }\end{array}$ & USA & $\begin{array}{l}\text { Psychiatric } \\
\text { hospital }\end{array}$ & $\begin{array}{l}\text { Psychiatric } \\
\text { diagnosis }\end{array}$ & $\begin{array}{l}\text { Not } \\
\text { reported }\end{array}$ & 127 & $39.3(14.9)$ & & & $2.5(4.2)$ & & & & & & \\
\hline $\begin{array}{l}\text { Daumit GL, } \\
2008 \text { [48] }\end{array}$ & RCT & USA & $\begin{array}{l}\text { Secondary } \\
\text { services }\end{array}$ & Schizophrenia & DSM IV & 1.125 & $40.7(11.1)$ & & & $8.5(7.4)$ & & & & & & \\
\hline $\begin{array}{l}\text { Dickerson FB, } \\
2013[49]\end{array}$ & RCT & USA & $\begin{array}{l}\text { Secondary } \\
\text { services }\end{array}$ & SMl & DSM IV & 291 & & & & & & & & & & \\
\hline $\begin{array}{l}\text { Druss BG, } \\
2010[50]\end{array}$ & $\mathrm{RCT}$ & USA & $\begin{array}{l}\text { Secondary } \\
\text { services }\end{array}$ & SMl & $\begin{array}{l}\text { Not } \\
\text { reported }\end{array}$ & 407 & & & & & & & & & & \\
\hline $\begin{array}{l}\text { Ferreira L, } \\
2010[51]\end{array}$ & Case-control & Portugal & $\begin{array}{l}\text { Secondary } \\
\text { services }\end{array}$ & Schizophrenia & DSM IV & 125 & $41.0(11.0)$ & & & & & 1,721 & $41.0(12.0)$ & & & \\
\hline $\begin{array}{l}\text { Foguet-Boreu } \\
\text { Q, } 2013 \text { [32] }\end{array}$ & $\begin{array}{l}\text { Cross- } \\
\text { sectional }\end{array}$ & Spain & $\begin{array}{l}\text { Secondary } \\
\text { services }\end{array}$ & SMl & $\begin{array}{l}\text { Not } \\
\text { reported }\end{array}$ & 137 & $51.1(12.9)$ & & & & & & & & & \\
\hline $\begin{array}{l}\text { Garcia-Portilla } \\
\text { MP, } 2009 \text { [52] }\end{array}$ & $\begin{array}{l}\text { Cross- } \\
\text { sectional }\end{array}$ & Spain & $\begin{array}{l}\text { Secondary } \\
\text { services }\end{array}$ & Bipolar disorders & ICD10 & 194 & 46.6 & & & $7.6(7.4)$ & $1.8(4.4)$ & & & & & \\
\hline $\begin{array}{l}\text { Goodrich DE, } \\
2012 \text { [53] }\end{array}$ & RCT & USA & $\begin{array}{l}\text { Secondary } \\
\text { services }\end{array}$ & Schizophrenia & $\begin{array}{l}\text { Not } \\
\text { reported }\end{array}$ & 134 & $52.8(9.9)$ & & & & & & & & & \\
\hline $\begin{array}{l}\text { Goff DC, } \\
2005 \text { [24] }\end{array}$ & Case-control & USA & $\begin{array}{l}\text { Secondary } \\
\text { services }\end{array}$ & Schizophrenia & DSM IV & 689 & $40.4(11.2)$ & & & & & 687 & $40.4(11.2)$ & & & 6.5 \\
\hline $\begin{array}{l}\text { Grover S, } \\
2014[54]\end{array}$ & $\begin{array}{l}\text { Cross- } \\
\text { sectional }\end{array}$ & India & Hospital & Bipolar disorder & ICD10 & 105 & $39.6(13.1)$ & & & $3.4(5.0)$ & $1.7(1.8)$ & & & & & \\
\hline $\begin{array}{l}\text { Hoffman BM, } \\
2010 \text { [55] }\end{array}$ & RCT & USA & H ospital & $\begin{array}{l}\text { Depressive } \\
\text { disorder }\end{array}$ & DSM IV & 46 & $53.4(7.0)$ & $\begin{array}{l}14.0(9.0) \\
\text { Only males. }\end{array}$ & & & & & & & & \\
\hline $\begin{array}{l}\text { Jin H, } 2011 \\
{[56]}\end{array}$ & $\begin{array}{l}\text { Cross- } \\
\text { sectional }\end{array}$ & USA & $\begin{array}{l}\text { Secondary } \\
\text { services }\end{array}$ & $\begin{array}{l}\text { With psychotic } \\
\text { symptoms }\end{array}$ & DSM IV & 179 & 63.1 & & & & & & & & & \\
\hline
\end{tabular}


Table 2 Characteristics of the studies included in the review (Continued)

\begin{tabular}{|c|c|c|c|c|c|c|c|c|c|c|c|c|c|c|c|}
\hline $\begin{array}{l}\text { Mackin P, } \\
2007[25]\end{array}$ & Case-control & UK & $\begin{array}{l}\text { Secondary } \\
\text { services }\end{array}$ & $\begin{array}{l}\text { Psychiatric } \\
\text { diagnosis }\end{array}$ & $\begin{array}{l}\text { Not } \\
\text { reported }\end{array}$ & 90 & $45.7(11.8)$ & $11.3(12.3)$ & $1.7(3.2)$ & $9.3(10.5)$ & 92 & 43.5 (13.6) & $6.8(6.4)$ & $1.0(1.1)$ & $4.7(4.3)$ \\
\hline $\begin{array}{l}\text { Margari F, } \\
2013 \text { [26] }\end{array}$ & $\begin{array}{l}\text { Cross- } \\
\text { sectional }\end{array}$ & Italy & $\begin{array}{l}\text { Psychiatric } \\
\text { hospital }\end{array}$ & $\begin{array}{l}\text { Psychiatric } \\
\text { diagnosis }\end{array}$ & DSM IV & 83 & $47.0(9.0)$ & $8.3(5.8)$ & & & 77 & $52.0(8.6)$ & $10.7(5.9)$ & & \\
\hline $\begin{array}{l}\text { McCreadie } \\
\text { RG, } 2003 \text { [27] }\end{array}$ & $\begin{array}{l}\text { Cross- } \\
\text { sectional }\end{array}$ & UK & $\begin{array}{l}\text { Secondary } \\
\text { services }\end{array}$ & Schizophrenia & DSM IV & 102 & $45.0(13.0)$ & & & $9.5(7.6)$ & & & & & $4.1(4.0)$ \\
\hline $\begin{array}{l}\text { McLean G, } \\
2014 \text { [23] }\end{array}$ & $\begin{array}{l}\text { Cross- } \\
\text { sectional }\end{array}$ & UK & Primary care & $\begin{array}{l}\text { Schizophrenia \& } \\
\text { related disorders }\end{array}$ & $\begin{array}{l}\text { Read } \\
\text { code }\end{array}$ & 1.942 & & & & & 215,165 & & & & \\
\hline $\begin{array}{l}\text { Nurjono M, } \\
2014[57]\end{array}$ & $\begin{array}{l}\text { Cross- } \\
\text { sectional }\end{array}$ & Singapore & $\begin{array}{l}\text { Psychiatric } \\
\text { hospital }\end{array}$ & Schizophrenia & DSM IV & 64 & & & & & & & & & \\
\hline $\begin{array}{l}\text { Osborn DP, } \\
2006 \text { [36] }\end{array}$ & $\begin{array}{l}\text { Cross- } \\
\text { sectional }\end{array}$ & UK & Primary care & SMI & $\begin{array}{l}\text { Not } \\
\text { reported }\end{array}$ & 74 & & & & & 148 & & & & \\
\hline $\begin{array}{l}\text { Protopopova } \\
\text { D, } 2012 \text { [58] }\end{array}$ & $\begin{array}{l}\text { Cross- } \\
\text { sectional }\end{array}$ & $\begin{array}{l}\text { Czech } \\
\text { Republic }\end{array}$ & $\begin{array}{l}\text { Psychiatric } \\
\text { hospital }\end{array}$ & $\begin{array}{l}\text { Schizophrenia \& } \\
\text { pychoses }\end{array}$ & ICD10 & 129 & 36.0 (11.9) & & & & & & & & \\
\hline $\begin{array}{l}\text { Ratliff JC, } \\
2013[28]\end{array}$ & Case-control & USA & $\begin{array}{l}\text { Secondary } \\
\text { services }\end{array}$ & $\begin{array}{l}\text { Schizophrenia \& } \\
\text { related disorders }\end{array}$ & DSM IV & 115 & $47.5(8.3)$ & 10.7 & & & 197 & $47.7(8.5)$ & 8.5 & & \\
\hline $\begin{array}{l}\text { Said MA, } \\
2012 \text { [59] }\end{array}$ & $\begin{array}{l}\text { Cross- } \\
\text { sectional }\end{array}$ & Malaysia & Hospital & Schizophrenia & DSM IV & 270 & & & & $6.3(5.6)$ & & & & & \\
\hline $\begin{array}{l}\text { Stroup TS, } \\
2013 \text { [60] }\end{array}$ & $\mathrm{RCT}$ & USA & $\begin{array}{l}\text { Secondary } \\
\text { services }\end{array}$ & $\begin{array}{l}\text { Schizophrenia \& } \\
\text { related disorders. }\end{array}$ & $\begin{array}{l}\text { Not } \\
\text { reported }\end{array}$ & 215 & $41.1(11.1)$ & & & $7.3(5.7)$ & & & & & \\
\hline $\begin{array}{l}\text { Sicras-Mainar } \\
\text { A, } 2013 \text { [61] }\end{array}$ & $\begin{array}{l}\text { Cross- } \\
\text { sectional }\end{array}$ & Spain & $\begin{array}{l}\text { Primary, } \\
\text { secondary \& } \\
\text { hospital care } \\
\text { services }\end{array}$ & $\begin{array}{l}\text { Schizophrenia \& } \\
\text { related disorders }\end{array}$ & DSM IV & 705 & $48.2(15.8)$ & $11.9(5.7)$ & & & & & & & \\
\hline $\begin{array}{l}\text { Slomka JM, } \\
2012 \text { [62] }\end{array}$ & $\mathrm{RCT}$ & USA & $\begin{array}{l}\text { Secondary } \\
\text { services }\end{array}$ & Bipolar disorder & $\begin{array}{l}\text { Not } \\
\text { reported }\end{array}$ & 118 & $53.0(9.9)$ & $13.7(10.0)$ & & & & & & & \\
\hline $\begin{array}{l}\text { Smith PJ, } \\
2007[63]\end{array}$ & $\mathrm{RCT}$ & USA & Not reported & $\begin{array}{l}\text { Depressive } \\
\text { disorder }\end{array}$ & DSM IV & 198 & $51.6(7.5)$ & & & $5.4(3.2)$ & & & & & \\
\hline $\begin{array}{l}\text { Tay YH, } 2013 \\
{[29]}\end{array}$ & $\begin{array}{l}\text { Cross- } \\
\text { sectional }\end{array}$ & China & $\begin{array}{l}\text { Secondary } \\
\text { services }\end{array}$ & Schizophrenia & DSM IV & 83 & $36.2(7.7)$ & $4.7(4.7)$ & & & 243 & $34.6(8.2)$ & $3.1(3.2)$ & & \\
\hline $\begin{array}{l}\text { Taylor V, } \\
2010 \text { [64] }\end{array}$ & Case-control & Canada & $\begin{array}{l}\text { Secondary } \\
\text { services }\end{array}$ & $\begin{array}{l}\text { Bipolar disorder \& } \\
\text { major depressive } \\
\text { disorder }\end{array}$ & DSM IV & 54 & $25.9(7.0)$ & & & & 104 & & & & \\
\hline $\begin{array}{l}\text { Wysokiński A, } \\
2012 \text { [65] }\end{array}$ & $\begin{array}{l}\text { Retrospective } \\
\text { review }\end{array}$ & Poland & $\begin{array}{l}\text { Psychiatric } \\
\text { hospital }\end{array}$ & $\begin{array}{l}\text { Psychotic } \\
\text { disorder }\end{array}$ & ICD10 & 62 & $38.0(12.4)$ & $6.4(7.2)$ & $3.7(2.8)$ & $5.8(6.1)$ & & & & & \\
\hline $\begin{array}{l}\text { Zuidersma M, } \\
2015 \text { [66] }\end{array}$ & $\begin{array}{l}\text { Cross- } \\
\text { sectional }\end{array}$ & Netherlands & $\begin{array}{l}\text { Primary \& } \\
\text { secondary care } \\
\text { services }\end{array}$ & $\begin{array}{l}\text { Depressive } \\
\text { disorder }\end{array}$ & DSM IV & 352 & $70.7(7.4)$ & $5.8(3.8)$ & & & & & & & \\
\hline
\end{tabular}

Abbreviations: FRS Framingham risk score, CVD cardiovascular disease, CHD cardiovascular heart disease, MI myocardial infarction, SCORE systematic coronary risk evaluation, NE: SMI severe mental illness, DSM-IV diagnostic and statistical manual of mental disorders, 4th Edition, ICD-10 International classification of diseases, 10th revision, RCT randomized controlled trial 
Table 3 Cardiovascular risk assessment by diagnostic group (depressive, bipolar, SMI and schizophrenia)

\begin{tabular}{|c|c|c|c|c|c|c|}
\hline \multirow[t]{3}{*}{ Diagnosis Groups } & \multirow[t]{3}{*}{ Author, year } & \multicolumn{4}{|c|}{ Psychiatric Group } & \multirow[t]{3}{*}{ Notes } \\
\hline & & \multicolumn{3}{|c|}{ FRS [mean (SD)] } & \multirow[t]{2}{*}{ SCORE [mean (SD)] } & \\
\hline & & CVD & Stroke & $\mathrm{CHD}$ & & \\
\hline \multirow{3}{*}{$\begin{array}{l}\text { Bipolar } \\
\text { disorder }\end{array}$} & Grover S, 2014 [54] & & & $3.4(5.0)$ & $1.7(1.8)$ & \\
\hline & Slomka JM, 2012 [62] & $13.7(10.0)$ & & & & \\
\hline & Garcia-Portilla MP, 2009 [52] & & & $7.6(7.4)$ & $1.8(4.4)$ & \\
\hline \multirow[t]{5}{*}{$\begin{array}{l}\text { Depressive } \\
\text { disorder }\end{array}$} & Acharya T, 2013 [43] & & & & & $\begin{array}{l}\text { FRS (CHD) expressed by types of } \\
\text { antidepressive medication groups. }\end{array}$ \\
\hline & Allan CL, 2011 [22] & & $10.3(7.6)$ & & & \\
\hline & Hoffman BM, 2010 [55] & $14.0(9.0)$ & & & & \\
\hline & Smith PJ, 2007 [63] & & & 3.2 & & \\
\hline & Zuidersma M, 2015 [66] & $5.8(3.8)$ & & & & \\
\hline \multirow[t]{13}{*}{ Schizophrenia } & Bernardo M, 2009 [45] & & & & & $\begin{array}{l}\text { SCORE: }<1 \%: 15.1 \% ; 1-4 \%: 68.8 \% ; 5-10 \% \text { : } \\
6.1 \% ; 11-15 \%: 0.3 \% \text { and } \geq 15 \% ; 0.1 \%\end{array}$ \\
\hline & Daumit GL, 2008 [48] & & & $8.5(7.4)$ & & \\
\hline & Ferreira L, 2010 [51] & & & & & $\begin{array}{l}\text { SCORE: no statistically significant difference } \\
\text { between case and controls was observed. }\end{array}$ \\
\hline & Goodrich DE, 2012 [53] & & & & & $\begin{array}{l}\text { FRS (CVD): }<10 \%: 40.7 \%, 10-20 \%: 40.7 \% \\
\text { and > } \% \text { \%: } 18.6 \%\end{array}$ \\
\hline & Goff DC, 2005 [24] & & & & & $\begin{array}{l}\text { FRS (CHD): In men: CATIE study: } 9.4 \text { (7.2); } \\
\text { NHANES study: } 7.0 \text { (6.3) and in women: } \\
6.3 \text { (6.3) and } 4.2 \text { (4.5), respectively. }\end{array}$ \\
\hline & McCreadie RG, 2003 [27] & & 4.1 & 9.6 & & \\
\hline & Nurjono M, 2014 [57] & & & & & $\begin{array}{l}\text { FRS (CVD): Participants in the highest } \\
\text { quartile of serum BDNF had a } 3.3 \text { times } \\
\text { increased in FRS over those in the } \\
\text { lowest quartile. }\end{array}$ \\
\hline & Said MA, 2012 [59] & & & $6.3(5.6)$ & & $\begin{array}{l}31.5 \% \text { of patients in the metabolic } \\
\text { syndrome group had a high/very high } \\
\text { FRS (CHD) vs. } 11 \% \text { in non-metabolic } \\
\text { syndrome group ( } p<0.001)\end{array}$ \\
\hline & Tay YH, 2013 [29] & $4.7(4.7)$ & & & & \\
\hline & Protopopova D, 2012 [58] & & & & & SCORE $\geq 5 \%: 10 \%$ \\
\hline & Arango C, 2008 [44] & & & $6.8(6.9)$ & $0.9(1.9)$ & \\
\hline & Cohn T, 2004 [37] & & & & & $\begin{array}{l}\text { FRS (MI): } 8.9 \% \text { in males, compared with } \\
\text { control subjects }(6.3)(p<0.001) \text { and } 2.6 \% \\
\text { females (vs. Control subjects } 2.0 \%)(p=0.180) \text {. }\end{array}$ \\
\hline & McLean G, 2014 [23] & & & & & $\begin{array}{l}\text { Joint British Societies score: risk levels by } \\
\text { age group and gender. Age was a major factor } \\
\text { being identified as high risk ( }>20 \%) \text {, with } 79 \% \\
\text { of those with schizophrenia aged } 65-74 \text { estimated } \\
\text { at high risk compared with only } 1.3 \% \text { of those } \\
\text { aged 35-44. }\end{array}$ \\
\hline
\end{tabular}

Ratliff JC, 2013 [28] $\quad 10.7$

Stroup TS, 2013 [60]

Sicras-Mainar A, 2013 [61] 11.9 (5.7)

SMI

Dickerson FB, 2013 [49]

Druss BG, 2010 [50]

Foguet-Boreu Q, 2013 [32]

FRS (CVD) in smokers 13.2 (11.9) and nonsmokers 7.4 (7.2)

FRS (CHD): 6.9 for intervention and 9.8 for control group.

FRS (REGICOR): high ( $\geq 10 \%$ ): $4.6 \%$ and SCORE: high ( $\geq 5 \%$ ): $5.4 \%$ 
Table 3 Cardiovascular risk assessment by diagnostic group (depressive, bipolar, SMI and schizophrenia) (Continued)

\begin{tabular}{|c|c|c|c|c|c|}
\hline & Osborn DP, 2006 [36] & & & & FRS (CHD): median: 5 \% (IQR:2-12) \\
\hline \multirow{7}{*}{$\begin{array}{l}\text { Psychiatric } \\
\text { diagnoses }\end{array}$} & Wysokiński A, 2012 [65] & $6.4(7.2)$ & $3.7(2.8)$ & $5.8(6.1)$ & \\
\hline & Correll CU, 2006 [46] & & & & $\begin{array}{l}\text { FRS (CHD): } 8.29(0.49) \text { in men and } 2.33(0.52) \\
\text { in women. }\end{array}$ \\
\hline & Jin H, 2011 [56] & & & & $\begin{array}{l}\text { FRS (CHD) was increased by } 79 \% \text { in } \\
\text { schizophrenia, } 72 \% \text { in posttraumatic } \\
\text { stress disorder and } 61 \% \text { in mood disorder. }\end{array}$ \\
\hline & Mackin P, 2007 [25] & $11.3(12.3)$ & $1.7(3.2)$ & $9.3(10.5)$ & \\
\hline & Margari F, 2013 [26] & $8.3(5.8)$ & & & \\
\hline & Correll CU, 2011 [47] & & & $2.5(4.2)$ & \\
\hline & Taylor V, 2010 [64] & & & & $\begin{array}{l}\text { FRS (CHD) was lower for patients at baseline } \\
\text { and follow-up, but increased across the follow-up } \\
\text { period ( } 2 \text {-years). Women patients showed an } \\
\text { increase risk for CHD over time, and men did not. }\end{array}$ \\
\hline
\end{tabular}

Data are expressed as mean (SD), unless otherwise stated

Abbreviations: FRS Framingham risk score, CVD cardiovascular disease, CHD cardiovascular heart disease, MI myocardial infarction, SCORE systematic coronary risk evaluation, SMI severe mental illness, PTSD posttraumatic stress disorder

\section{Discussion}

Data from studies that reported CVR scores did not support higher risk in patients with SMI than in the control population. Subgroup analysis showed a higher CVR associated with schizophrenia than with other SMIs. Only in patients with schizophrenia was there some evidence of higher CVR scores than in the control population.

To date it has been widely accepted that the prevalence of modifiable risk factors was increased in patients

Table 4 Stratified pooled standardized mean differences for cardiovascular risk assessment

\begin{tabular}{llll}
\hline & Number of studies & $\begin{array}{l}\text { SMD } \\
(95 \% \mathrm{Cl})\end{array}$ & $R^{2}$ \\
\hline Diagnosis & & $0.03(-0.48,0.54)$ & $0 \%$ \\
Depressive disorder & 1 & $0.63(0.16,1.09)$ & $94 \%$ \\
Schizophrenia & 3 & $0.02(-0.82,0.86)$ & $92 \%$ \\
Psychiatric diagnosis & 2 & & \\
Criteria for diagnosis & & $0.35(0.24,0.45)$ & $84 \%$ \\
NE & 1 & $0.32(-0.11,0.75)$ & $94 \%$ \\
DSM IV & 5 & $0.34(-0.21,0.88)$ & $94 \%$ \\
Study design & & $0.35(0.24,0.45)$ & $0 \%$ \\
Observational study & 5 & & \\
RCT & 1 & $0.40(-0.44,1.02)$ & $95 \%$ \\
Outcome & & $0.35(0.24,0.45)$ & $0 \%$ \\
CVD total & 4 & $0.03(-0.48,0.54)$ & $0 \%$ \\
CHD & 1 & 1 &
\end{tabular}

Abbreviations: SMD standardized mean difference, $\mathrm{Cl}$ confidence interval, $N E$ non-specific, DSM IV statistical manual of mental disorders, $R C T$ randomized controlled trial, CVD cardiovascular disease, CHD coronary heart disease with SMI [30]. Nonetheless, several authors have suggested that not all risk factors were equally increased in these patients. A number of studies have found that smoking and diabetes rates were higher in the SMI population than in the reference population [31], while others observed that hypertension was not increased among SMI patients [31-33]. Conversely, other authors did not detect significant differences in CVR factors between participants with and without SMI [34]. This could be the result of publication bias affecting CVR studies and therefore affecting CVR assessment. Chapman et al., in a meta-analysis of 42 studies on smoking in patients with schizophrenia, revealed that studies reporting low prevalence of this risk factor are cited less often than those reporting higher prevalence in this population [35].

Numerous previous studies have noted the importance of CVR factors in patients with SMI, but only a few of them incorporated CVR evaluation in the last 12 years. No other systematic review has been found in the literature on this topic. However, Osborn et al., in a systematic review which objective was to determine the relative risk of some CVR factors in people with SMIs, synthesized data about some studies that reported 10 year cardiovascular risk scores [31]. One controlled community study, including 74 SMI patients found that excess CVR scores showed that participants with SMI had higher FRS (CHD) than participants without SMI (median 10year risk: 5 vs. $4 \%$ ) [36]. Another study, including 84 schizophrenic patients showed a significant increase of CVR only in males based on FRS (CHD) (10.4 vs. 6.4 \%) [27]. And the last, involves 240 patients schizophrenia and schizoaffective disorders showed also an increased risk based on FRS (MI) score only in male patients compared to controls (8.9 vs. $6.3 \%$ ) [37]. Our review also 


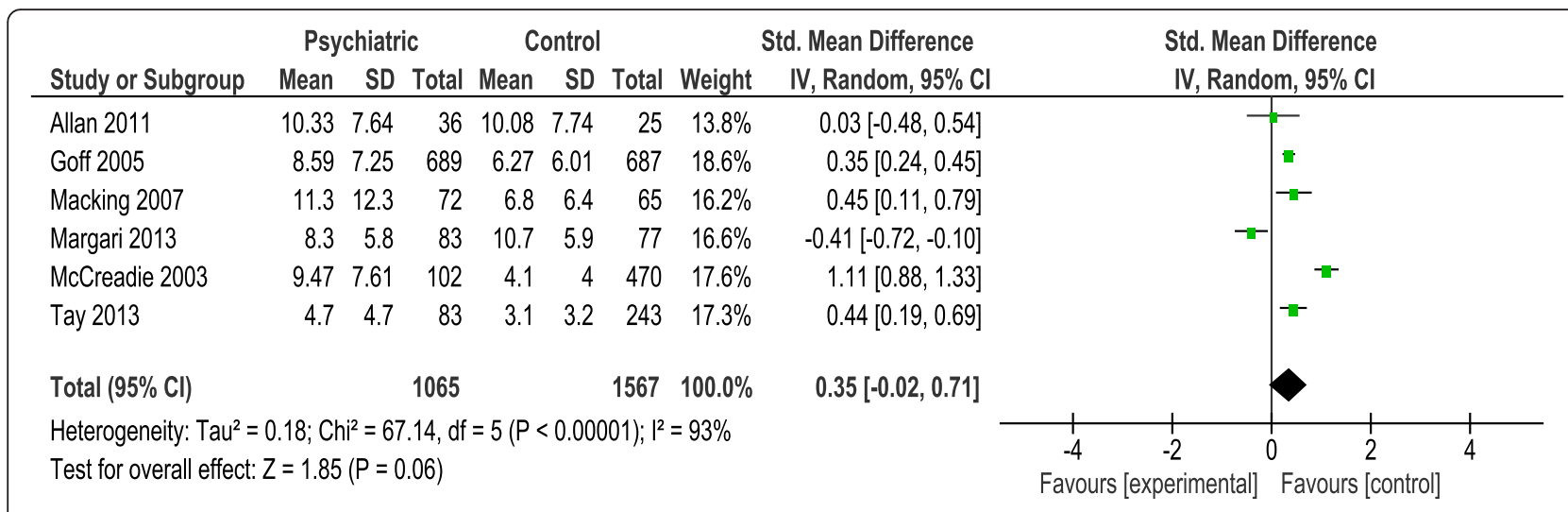

Fig. 2 Summary of forest plot. Standardized mean differences between psychiatric group and comparative group

showed that schizophrenia is the group that have more evidence of higher CVR than control groups and is consistent with other studies [38, 39].

However, the discrepancy between data showing higher CVR in SMI and the CVR assessment obtained in our review raises some questions. The tools to measure CVR that have been validated for general population may not apply to patients with SMI. In this sense, Osborn et al. proposed a CVR prediction model for this population [40]. In addition to the usual predictors, this model also included social deprivation, heavy alcohol use, SMI diagnosis, and prescriptions for antidepressants and antipsychotics [40]. Another key point is the influence of the prescribed medications on CVR. There is strong evidence that antipsychotic drugs, and to a more restricted degree antidepressants and mood stabilizers, are associated with an increased risk for several physical diseases, including obesity, dyslipidaemia, diabetes mellitus and so on [41]. Furthermore, unclear benefits of different kinds of antipsychotics (first vs. second-generation antipsychotics) have been reported [42], despite the superior efficacy and greater treatment persistence attributed to second-generation antipsychotics.

Our analysis showed that schizophrenia is the group at highest risk, in consonance with other studies that showed an increased risk in patients with schizophrenia and depression, compared to other SMIs [41, 42]. Of the three studies of schizophrenic patients included in the forest plot summary, McCreadie et al. [27] clearly had the highest SMD. This difference may be explained by the inclusion of older patients with a longer history of illness compared to the other two studies [24, 29].

\section{Strengths and weaknesses of this review}

The major strength of this study is that it is the first review to focus on CVR assessments in patients with SMI. The search identified a large number of studies (67) that showed CVR data. Osborn et al. centred their attention on studies of CVR factors and also showed results of CVR assessments provided by 4 studies, three of which included only schizophrenia and related disorders; one study also included nonaffective chronic psychotic illness [31].

Our study also has a number of limitations to be taken into account. We only searched a single data source, although this limitation was countered by an extensive manual search (snowball method). Furthermore, a large number of studies had no control group, making it impossible to include them in the meta-analysis. Of the 7 studies with control groups [22, 24-29], only 6 had sufficient data for inclusion in the meta-analysis and the heterogeneity of data synthesis was considerable $\left(\mathrm{I}^{2}>75 \%\right)$. Therefore, all the conclusions of the meta-analysis should be considered with caution. The variability of the studies included in the meta-analysis could be attributed to the ages of the participants, the diagnoses included, and differences in study design.

\section{Implications for future research}

Further work is needed to establish whether patients with SMI have increased CVR compared to general population. More information on the type of CVR assessment used would help to establish a greater degree of accuracy on this question. A new risk assessment approach may be needed in future studies in order to include other relevant factors (obesity, psychotropic drugs and social deprivation) [38]. In addition, a discussion is needed to reach consensus on an operational definition of SMI that can be applied for research purposes.

\section{Conclusions}

A review of literature reporting CVR assessment in patients with SMI did not find sufficient evidence to determine whether or not there is a higher risk in these patients relative to the reference population. Subgroup 
analysis showed a higher CVR in patients with schizophrenia compared to those with depressive disorder or a psychiatric diagnosis. Only in patients with schizophrenia was there some evidence of higher CVR scores than in the control population. Instead of the generalized idea that SMI is associated with increased CVR, it is important to consider the complexity of summarizing the data because there is no universal definition of SMI or standard methods to describe CVR in this population. Further work is needed to elucidate whether new CVR charts that incorporate intrinsic determinants (as the effect of psychotropic drugs or social deprivation) should be established for risk assessment in this population.

\section{Ethics and consent to participate}

Not Applicable.

\section{Consent to publish}

Not Applicable.

\section{Availability of data and materials}

The data and materials used in this review are available on request.

\section{Additional files}

Additional file 1: Appendix 1. Quality assessment of the observational studies retained in the Review (STROBE). Quality assessment of the clinical trials studies retained in the Review (CONSORT). (DOC $97 \mathrm{~kb}$ )

Additional file 2: Appendix 2. Funnel plot. (JPG $26 \mathrm{~kb}$ )

\section{Abbreviations}

CHD: cardiovascular heart disease; CVD: cardiovascular disease; CVR: cardiovascular risk; DSM-IV: diagnostic and statistical manual of mental disorders, 4th edition; FRS: Framingham risk score; ICD-10: International Classification of Diseases, 10th revision; Ml: myocardial infarction; SCORE: systematic coronary risk evaluation; SMl: severe mental illness; WHO: World Health Organization.

\section{Competing interests}

All authors have completed the ICMJE uniform disclosure form at www.icmje.org/coi_disclosure.pdf (available on request from the corresponding author) and declare: no support from any organisation for the submitted work; no financial relationships with any organisations that might have an interest in the submitted work in the previous three years; no other relationships or activities that could appear to have influenced the submitted work.

\section{Authors' contributions}

QFB and MFS were responsible for the study concept and design, wrote the review protocol, supervised and carried out the data extraction. QFB and EZO designed and performed the search strategies for the electronic databases. QFB, CVF, MFS LMM, JMH, LAG and MPP collaborated in the data extraction. GFM contributed to the analysis and interpretation of the data and designed the meta-analysis. QFB and CVF reviewed articles for inclusion, carried out the data extraction, and contributed to the analysis, interpretation and presentation of data. QFB drafted the manuscript with the input of the other authors. All authors contributed a critical revision of the manuscript and had full access to all of the data (including statistical reports and tables) and take responsibility for the integrity of the data and the accuracy of the data analysis. QFB is guarantor. All authors read and approved the final manuscript.

\section{Acknowledgements}

The authors appreciate the English language review by Elaine Lilly, PhD, and are grateful to Carmen Ibáñez for administrative support.

\section{Funding}

This research has been funded by the Ministry of Science and Innovation through the Instituto Carlos III (PI12/00427). This study received no specific grant from any funding agency in the public, commercial, or not-for-profit sectors. LA is funded by the National Institute of Health Research (NIHR) UK. The funders had no role in the study design, collection, analysis and interpretation of data, writing of the manuscript and decision to submit for publication. The content is solely the responsibility of the authors and does not necessarily represent the official views of IDIAP Jordi Gol and NIHR.

\section{Author details}

'Institut Universitari d'Investigació en Atenció Primària Jordi Gol (IDIAP Jordi Gol), Universitat Autònoma de Barcelona, Gran Via Corts Catalanes, 587 àtic, 08007 Barcelona, Spain. ${ }^{2}$ Department of Medical Sciences, School of Medicine, University of Girona, Emili Grahit, 77, 17071 Girona, Spain. ${ }^{3}$ Técnica de Salud ICS, Unitat Docent AFiC, Sardenya, 375, Entl., 08025 Barcelona, Spain. ${ }^{4}$ Faculty of Nursing, University of Girona, Emili Grahit, 77, 17071 Girona, Spain. ${ }^{5}$ The Westborough Road Health Centre, 258 Westborough Road, Westcliff-on-Sea SSO 9PT, United Kingdom. ${ }^{6}$ Centre of Primary Care and Public Health, Queen Mary University of London, Yvone Carter Building 58 Tuner Street, E1 2AB London, United Kingdom. ${ }^{7}$ Departamento de Psiquiatría y Medicina Legal, Instituto de Neuropsiquiatría y Adicciones (INAD), Hospital del Mar Parc de Salut Mar., Universidad Autónoma de Barcelona, Passeig Marítim 25-29, 08003 Barcelona, Spain. ${ }^{8}$ Gimbernat School of Nursing, Universitat Autònoma de Barcelona, Avinguda de la Generalitat, 202-206, Sant Cugat del Vallès, 08174 Barcelona, Spain.

Received: 29 June 2015 Accepted: 25 April 2016

Published online: 12 May 2016

\section{References}

1. Lozano R, Naghavi M, Foreman K, Lim S, Shibuya K, Aboyans V, et al. Global and regional mortality from 235 causes of death for 20 age groups in 1990 and 2010: a systematic analysis for the Global Burden of Disease Study 2010. Lancet. 2012;380(9859):2095-128.

2. Murray CJ, Vos T, Lozano R, Naghavi M, Flaxman AD, Michaud C, et al. Disability-adjusted life years (DALYs) for 291 diseases and injuries in 21 regions, 1990-2010: a systematic analysis for the Global Burden of Disease Study 2010. Lancet. 2012;380(9859):2197-223.

3. American Psychiatric Association. Diagnostic and statistical manual of mental disorders. Fifth. Arlington: American Psychiatic Publishing; 2013.

4. Ruggeri M, Leese M, Thornicroft G, Bisoffi G, Tansella M. Definition and prevalence of severe and persistent mental illness. Br J Psychiatry. 2000;177: 149-55.

5. Guideline development group of the Clinical Practice Guideline on Psychosocial Interventions in Severe Mental Illness. Clinical practice guideline on psychosocial interventions in severe mental illness. Madrid: Ministry of Health and Social Policy; 2009.

6. De Hert M, Correll CU, Bobes J, Cetkovich-Bakmas M, Cohen D, Asai I, et al. Physical illness in patients with severe mental disorders. I. Prevalence, impact of medications and disparities in health care. World Psychiatry. 2011; 10:52-77.

7. Newcomer JW. Medical risk in patients with bipolar disorder and schizophrenia. J Clin Psychiatry. 2006;67:e16.

8. Pérez-Piñar M, Mathur R, Foguet Q, Ayis S, Robson J, Ayerbe L. Cardiovascular risk factors among patients with schizophrenia, bipolar depressive, anxiety, and personality disorders. Eur Psychiatry. 2016;35:8-15.

9. Mainar A, Blanca-Tamayo M, Rejas-Gutiérrez J, Navarro- Artieda R. Metabolic syndrome in outpatients receiving antipsychotic therapy in routine clinical practice: a crosssectional assessment of a primary health care database. Eur Psychiatry. 2008;23:100-8.

10. Jomas BS, Franks P, Ingram DD. Are symptoms of anxiety and depression risk factors for hypertension? Longitudinal evidence from the National Health and Nutrition Examination Survey I Epidemiologic Follow-up Study. Arch Fam Med. 1997;6:43-9. 
11. Patten SB, Williams JV, Lavorato DH, Modgill G, Jetté N, Eliasziw M. Major depression as a risk factor for chronic disease incidence: longitudinal analyses in a general population cohort. Gen Hosp Psychiatry. 2008;30:407-11.

12. Gold KJ, Kilbourne AM, Valenstein M. Primary care of patients with serious mental illness: your chance to make a difference. J Fam Pract. 2008;57:515-25.

13. Bobes J, Sáiz J, Montes JM, Mostaza J, Rico-Villademoros F, Vieta E. Consenso Español de Salud Física del Paciente con Trastorno bipolar. Rev Psiquatr Salud Ment (Barc). 2008;1:26-37.

14. Ruetsch O, Viala A, Bardou H, Martin P, Vacheron MN. Psychotropic drugs induced weight gain: a review of the literature concerning epidemiological data, mechanisms and management. Encéphale. 2005;31(4 Pt 1):507-16.

15. Taylor D. Antidepressant drugs and cardiovascular pathology: a clinical overview of affectiveness and safety. Acta Psychiatr Scand. 2008;118:434-42.

16. Kaplan NM. Clinical hypertension. 5th ed. Madrid: Lippincott Williams\&Wilkins; 2006.

17. Stroup DF, Berlin JA, Morton SC, Olkin I, Williamson GD, Rennie D, et al. Meta-analysis of observational studies in epidemiology: a proposal for reporting. Meta-analysis of observational studies in epidemiology (MOOSE) group. JAMA. 2000;283:2008-12.

18. Moher D, Liberati A, Tetzlaff J, Altman DG, The PRISMA Group. Preferred reporting items for systematic reviews and meta-analyses: the PRISMA statement. PLoS Med. 2009;6(6), e1000097.

19. Vandenbroucke JP, von Elm E, Altman DG, Gøtzsche PC, Mulrow CD, Pocock SJ, et al. Strengthening the Reporting of Observational Studies in Epidemiology (STROBE): explanation and elaboration. PLoS Med. 2007;4(10):e297.

20. Schulz KF, Altman DG, Moher D, CONSORT Group. CONSORT 2010 Statement: updated guidelines for reporting parallel group randomised trials. BMC Med. 2010;8:18.

21. Higgins JP, Thompson SG. Quantifying heterogeneity in a meta-analysis. Stat Med. 2002:21(11):1539-58.

22. Allan CL, Sexton CE, Kalu UG, McDermott LM, Kivimäki M, Singh-Manoux A et al. Does the Framingham Stroke Risk Profile predict white-matter changes in late-life depression? Int Psychogeriatr. 2012;24(4):524-31.

23. McLean G, Martin JL, Martin DJ, Guthrie B, Mercer SW, Smith DJ. Standard cardiovascular disease risk algorithms underestimate the risk of cardiovascular disease in schizophrenia: evidence from a national primary care database. Schizophr Res. 2014;159(1):176-81.

24. Goff DC, Sullivan LM, McEvoy JP, Meyer JM, Nasrallah HA, Daumit GL, et al. A comparison of ten-year cardiac risk estimates in schizophrenia patients from the CATIE study and matched controls. Schizophr Res. 2005;80(1):45-53.

25. Mackin P, Bishop D, Watkinson H, Gallagher P, Ferrier IN. Metabolic disease and cardiovascular risk in people treated with antipsychotics in the community. Br J Psychiatry. 2007;191:23-9.

26. Margari F, Lozupone M, Pisani R, Pastore A, Todarello O, Zagaria G, et al. Metabolic syndrome: differences between psychiatric and internal medicine patients. Int J Psychiatry Med. 2013;45(3):203-26.

27. McCreadie RG, Scottish Schizophrenia Lifestyle Group. Diet, smoking and cardiovascular risk in people with schizophrenia: descriptive study. Br J Psychiatry. 2003;183:534-9.

28. Ratliff JC, Palmese LB, Reutenauer EL, Srihari VH, Tek C. Obese schizophrenia spectrum patients have significantly higher 10-year general cardiovascular risk and vascular ages than obese individuals without severe mental illness. Psychosomatics. 2013;54(1):67-73.

29. Tay $\mathrm{YH}$, Nurjono M, Lee J. Increased Framingham 10-year CVD risk in Chinese patients with schizophrenia. Schizophr Res. 2013;147(1):187-92.

30. De Hert M, Dekker JM, Wood D, Kahl KG, Holt Rl, Moller HJ. Cardiovascular disease and diabetes in people with severe mental illness position statement from the European Psychiatric Association (EPA), supported by the European Association for the Study of Diabetes (EASD) and the European Society of Cardiology (ESC). Eur Psychiatry. 2009;24:412-24.

31. Osborn DP, Wright CA, Levy G, King MB, Deo R, Nazareth I. Relative risk of diabetes, dyslipidaemia, hypertension and the metabolic syndrome in people with severe mental illnesses: systematic review and metaanalysis. BMC Psychiatry. 2008:8:84.

32. Foguet Boreu Q, Roura Poch P, Bullón Chia A, Mauri Martin C, Gordo Serra $\mathrm{N}$, Costa RC; en representación del grupo de Riesgo Cardiovascular en Trastorno Mental Severo (RISCA-TMS). Cardiovascular risk factors, cardiovascular risk and quality of life in patients with a severe mental disorder. Aten Primaria. 2013;45(3):141-8.

33. Castillo-Sánchez M, Fàbregas-Escurriola M, Bergè-Baquero D, Foguet-Boreu Q, Fernández-San Martín Ml, Goday-Arno A. Schizophrenia, antipsychotic drugs and cardiovascular risk: Descriptive study in primary care. Eur Psychiatry. 2015;30(4):535-41.

34. Clerici M, Bartoli F, Carretta D, Crocamo C, Bebbington P, Carrà G. Cardiovascular risk factors among people with severe mental illness in Italy: a cross-sectional comparative study. Gen Hosp Psychiatry. 2014;36(6):698-702.

35. Chapman S, Ragg M, McGeechan K. Citation bias in reported smoking prevalence in people with schizophrenia. Aust N Z J Psychiatry. 2009;43(3):277-82.

36. Osborn DP, Nazareth I, King MB. Risk for coronary heart disease in people with severe mental illness: cross-sectional comparative study in primary care. Br J Psychiatry. 2006;188:271-7.

37. Cohn T, Prud'homme D, Streiner D, Kameh H, Remington G. Characterizing coronary heart disease risk in chronic schizophrenia: high prevalence of the metabolic syndrome. Can J Psychiatry. 2004;49(11):753-60.

38. Fan Z, Wu Y, Shen J, Ji T, Zhan R. Schizophrenia and the risk of cardiovascular diseases: a meta-analysis of thirteen cohort studies. J Psychiatr Res. 2013;47(11): 1549-56.

39. Van der Kooy K, van Hout H, Marwijk H, Marten H, Stehouwer C, Beekman A. Depression and the risk for cardiovascular diseases: systematic review and metaanalysis. Int J Geriatr Psychiatry. 2007;22(7):613-26.

40. Osborn DP, Hardoon S, Omar RZ, Holt RI, King M, Larsen J, et al. Cardiovascular risk prediction models for people with severe mental illness: results from the prediction and management of cardiovascular risk in people with severe mental illnesses (PRIMROSE) research program. JAMA Psychiatry. 2015;72(2):143-51.

41. Correll CU, Detraux J, De Lepeleire J, De Hert M. Effects of antipsychotics, antidepressants and mood stabilizers on risk for physical diseases in people with schizophrenia, depression and bipolar disorder. World Psychiatry. 2015; 14(2):119-36

42. Hartling L, Abou-Setta AM, Dursun S, Mousavi SS, Pasichnyk D, Newton AS. Antipsychotics in adults with schizophrenia: comparative effectiveness of first-generation versus second-generation medications: a systematic review and meta-analysis. Ann Intern Med. 2012;157(7):498-511.

43. Acharya T, Acharya S, Tringali S, Huang J. Association of antidepressant and atypical antipsychotic use with cardiovascular events and mortality in a veteran population. Pharmacotherapy. 2013;33(10):1053-61.

44. Arango C, Bobes J, Kirkpatrick B, Garcia-Garcia M, Rejas J. Psychopathology, coronary heart disease and metabolic syndrome in schizophrenia spectrum patients with deficit versus non-deficit schizophrenia: findings from the CLAMORS study. Eur Neuropsychopharmacol. 2011;21(12):867-75.

45. Bernardo M, Cañas F, Banegas JR, Casademont J, Riesgo Y, Varela C; RICAVA Study Group. Prevalence and awareness of cardiovascular risk factors in patients with schizophrenia: a cross-sectional study in a low cardiovascular disease risk geographical area. Eur Psychiatry. 2009;24(7):431-41.

46. Correll CU, Frederickson AM, Kane JM, Manu P. Metabolic syndrome and the risk of coronary heart disease in 367 patients treated with secondgeneration antipsychotic drugs. J Clin Psychiatry. 2006;67(4):575-83.

47. Correll CU, Kane JM, Manu P. Obesity and coronary risk in patients treated with second-generation antipsychotics. Eur Arch Psychiatry Clin Neurosci. 2011;261(6):417-23.

48. Daumit GL, Goff DC, Meyer JM, Davis VG, Nasrallah HA, McEvoy JP, Rosenheck R, Davis SM, Hsiao JK, Stroup TS, Lieberman JA. Antipsychotic effects on estimated 10-year coronary heart disease risk in the CATIE schizophrenia study. Schizophr Res. 2008;105(1-3):175-87.

49. Dickerson FB, Yu A, Dalcin A, Jerome GJ, Gennusa 3rd JV, Charleston J, et al. Cigarette smoking and health characteristics in individuals with serious mental illness enrolled in a behavioral weight loss trial. J Dual Diagn. 2013;9(1):39-46.

50. Druss BG, von Esenwein SA, Compton MT, Rask KJ, Zhao L, Parker RM. A. The primary care access, referral, and evaluation (PCARE) study. Am J Psychiatry. 2010;167(2):151-931.

51. Ferreira L, Belo A, Abreu-Lima C. A case-control study of cardiovascular risk factors and cardiovascular risk among patients with schizophrenia in a country in the low cardiovascular risk region of Europe. Rev Port Cardiol. 2010;29(10):1481-93.

52. Garcia-Portilla MP, Saiz PA, Bascaran MT, Martínez AS, Benabarre A, Sierra P, et al. Cardiovascular risk in patients with bipolar disorder. J Affect Disord. 2009;115(3):302-8

53. Goodrich DE, Kilbourne AM, Lai Z, Post EP, Bowersox NW, Mezuk B, et al. Design and rationale of a randomized controlled trial to reduce cardiovascular disease risk for patients with bipolar disorder. Contemp Clin Trials. 2012;33(4):666-78 
54. Grover S, Nebhinani N, Chakrabarti S, Avasthi A, Basu D, Kulhara P, et al. Cardiovascular risk factors among bipolar disorder patients admitted to an inpatient unit of a tertiary care hospital in India. Asian J Psychiatr. 2014;10:51-5.

55. Hoffman BM, Sherwood A, Smith PJ, Babyak MA, Doraiswamy PM, Hinderliter A, et al. Cardiovascular disease risk, vascular health and erectile dysfunction among middle-aged, clinically depressed men. Int J Impot Res. 2010;22(1):30-5.

56. Jin H, Folsom D, Sasaki A, Mudaliar S, Henry R, Torres M, et al. Increased Framingham 10-year risk of coronary heart disease in middle-aged and older patients with psychotic symptoms. Schizophr Res. 2011;125(2-3):295-9.

57. Nurjono M, Tay $\mathrm{YH}$, Lee J. The relationship between serum brain-derived neurotrophic factor (BDNF) and cardiometabolic indices in schizophrenia. Schizophr Res. 2014;157(1-3):244-8.

58. Protopopova D, Masopust J, Maly R, Valis M, Bazant J. The prevalence of cardiometabolic risk factors and the ten-year risk of fatal cardiovascular events in patients with schizophrenia and related psychotic disorders. Psychiatr Danub. 2012;24(3):307-13.

59. Said MA, Sulaiman AH, Habil MH, Das S, Bakar AK, Yusoff RM, et al Metabolic syndrome and cardiovascular risk among patients with schizophrenia receiving antipsychotics in Malaysia. Singapore Med J. 2012; 53(12):801-7.

60. Stroup TS, Byerly MJ, Nasrallah HA, Ray N, Khan AY, Lamberti JS, et al. Effects of switching from olanzapine, quetiapine, and risperidone to aripiprazole on 10-year coronary heart disease risk and metabolic syndrome status: results from a randomized controlled trial. Schizophr Res. 2013;146(1-3):190-5.

61. Sicras-Mainar A, Rejas-Gutiérrez J, Navarro-Artieda R, Blanca-Tamayo M. Creactive protein as a marker of cardiovascular disease in patients with a schizophrenia spectrum disorder treated in routine medical practice. Eur Psychiatry. 2013;28(3):161-7.

62. Slomka JM, Piette JD, Post EP, Krein SL, Lai Z, Goodrich DE, et al. Mood disorder symptoms and elevated cardiovascular disease risk in patients with bipolar disorder. J Affect Disord. 2012;138(3):405-8.

63. Smith PJ, Blumenthal JA, Babyak MA, Hoffman BM, Doraiswamy PM, Waugh $\mathrm{R}$, et al. Cerebrovascular risk factors, vascular disease, and neuropsychological outcomes in adults with major depression. Psychosom Med. 2007;69(6):578-86.

64. Taylor V, McKinnon MC, Macdonald K, Jaswal G, Macqueen GM. Adults with mood disorders have an increased risk profile for cardiovascular disease within the first 2 years of treatment. Can J Psychiatry. 2010;55(6):362-8.

65. Wysokiński A, Kowman M, Kłoszewska I. The prevalence of metabolic syndrome and Framingham cardiovascular risk scores in adult inpatients taking antipsychotics - a retrospective medical records review. Psychiatr Danub. 2012;24(3):314-22.

66. Zuidersma M, Izaks GJ, Naarding P, Comijs HC, Oude Voshaar RC. Vascular burden and cognitive function in late-life depression. Am J Geriatr Psychiatry. 2015;23(5):514-24

\section{Submit your next manuscript to BioMed Central and we will help you at every step:}

- We accept pre-submission inquiries

- Our selector tool helps you to find the most relevant journal

- We provide round the clock customer support

- Convenient online submission

- Thorough peer review

- Inclusion in PubMed and all major indexing services

- Maximum visibility for your research

Submit your manuscript at www.biomedcentral.com/submit

) Biomed Central 